\title{
El rol de las tareas realistas en la interpretación del residuo de la división aritmética
}

The role of realistic tasks in interpreting the remainder of arithmetic division

\section{Lucía Zapata-Cardona *}

(iD) https://orcid.org/0000-0003-4266-5273

Tipo de Artículo: Informes de Investigación y ensayos inéditos

Doi: 10.17533/udea.unipluri.20.2.04

Zapata-Cardona, L. (2020). El rol de las tareas realistas en la interpretación del residuo de la división aritmética. Uni-Pluriversidad, 20(2). e2020204. doi: 10.17533/udea.unipluri.20.2.04

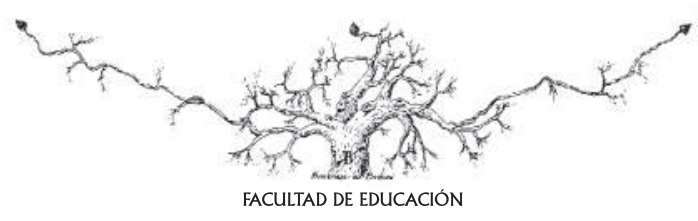

Recibido: 2020-03-25 • Aprobado: 2020-11-09

* Universidad de Antioquia. Colombia

Email: lucia.zapata1@udea.edu.co 


\title{
Resumen
}

Este artículo reporta una experiencia en la que se estudió el aporte de las tareas realistas inspiradas en la Educación Matemática Realista a la comprensión del residuo en la división aritmética en población infantil. Las participantes fueron tres estudiantes de la escuela primaria ( 6 a 10 años de edad) quienes resolvieron una tarea de división con residuo en un contexto realista. Las participantes fueron entrevistadas en un espacio por fuera del ambiente escolar y se les aportó manipulativos como apoyo a la solución y como herramienta para hacer visible las estrategias usadas en el modelado. Los principales hallazgos revelan que las tareas matemáticas realistas tienen el potencial para promover la exploración, construcción e implementación de algoritmos espontáneos en los estudiantes, parecen solucionar el problema de la interpretación del residuo en la división aritmética y promueven el modelado y la matematización dando acceso a una matemática mucho más formal.

Palabras clave: Matemática realista, aritmética escolar, infancia, división

\begin{abstract}
This paper reports an experience that studied the contribution of realistic tasks inspired by the Realistic Mathematics Education to the interpretation of the remainder in the arithmetic division in young children. The participants were three primary school students (6 to 10 years old) who solved a division task with remainder in a realistic context. The participants were interviewed in a space outside the school environment and they were provided with manipulatives to support the solution and as a tool to make visible the strategies used in the modeling. The main findings reveal that realistic mathematical tasks have the potential to promote exploration, construction, and implementation of spontaneous algorithms in students; seem to solve the problem of interpreting the remainder in arithmetic division; and promote modeling and mathematization by giving access to much more formal mathematics.
\end{abstract}

Keywords: Realistic mathematics, school arithmetic, childhood, division 


\section{INTRODUCCIÓN}

Tradicionalmente, el currículo de matemáticas en la escuela primaria ha buscado que los estudiantes aprendan operaciones aritméticas básicas (ver por ejemplo los Estándares Básicos de Competencias de Colombia [Ministerio de Educación Nacional, 2006] y The Common Core State Standard Initiative -Iniciativa de los Estándares Básicos Comunes de Estado- de los Estados Unidos [National Governors Association and Council of Chief State School Officers, 2010]). No obstante, la investigación ha mostrado que, en su paso por la educación obligatoria, los estudiantes aprenden un vasto cúmulo de conocimientos matemáticos a los que son incapaces de darle un buen uso (Freudenthal, 1991) y no logran conectar con escenarios por fuera de la escuela (Bakker y Derry, 2011; Zapata-Cardona, 2018).

Este problema podría estar relacionado con la forma en la que tradicionalmente se ha llevado a cabo la enseñanza de las matemáticas, que pone un fuerte énfasis en la solución de ejercicios rutinarios; el uso de problemas matemáticos restrictivos en los libros de texto; la fragmentación del conocimiento en partes sin sentido (van den Heuvel-Panhuizen, 2002); y la visión mecanicista de la matemática, que asume al hombre como un instrumento similar a una computadora que puede ser programado mediante la ejercitación para llevar a cabo operaciones matemáticas (Freudenthal, 1991). La literatura ha mostrado que la matemática escolar en la mayoría de los países hace un mayor énfasis en el dominio de reglas matemáticas básicas que en la comprensión (Vos, 2020) y que los libros de texto privilegian los procedimientos por encima de las matemáticas subyacentes (Zapata-Cardona y Marrugo, 2019). Al respecto, van den Heuvel-Panhuizen (2020) señala que los libros de texto de la escuela primaria en su mayoría contienen problemas de cálculo sencillos, pero las oportunidades para la resolución de problemas y el razonamiento matemático son muy limitadas. Una preocupación similar fue señalada por Gravemeijer (2020), quien indicó que los libros de texto privilegian procedimientos que generan rápidamente respuestas correctas y se invierte poco en el desarrollo matemático. Esto hace que la aritmética que aprenden los estudiantes de la escuela primaria se torne poco relevante y poco funcional. El siglo XXI requiere solucionadores de problemas y equipar a los estudiantes con una base sólida de conocimiento aritmético contextual parece un punto de partida valioso (Kool, 2020).

Algunos autores (Cisternas Rojas, Gil Llario, Ceccato, y Marí-Sanmillán, 2019) han señalado que los estudiantes tienden a confundir las operaciones aritméticas debido a la baja capacidad para reconocer el proceso necesario y la falta de procesamiento adecuado de la información. Otros autores (Kaasila, Pehkonen y Hellinen, 2010) han establecido que la comprensión de la división requiere una buena comprensión de la multiplicación. No obstante, el problema va más allá de los aspectos técnicos de la aritmética y también tiene que ver con las formas de presentar la aritmética escolar en la infancia. 
Se tiene la falsa creencia de que una fuerte formación aritmética será suficiente para que el aprendiz haga espontáneamente la conexión con el mundo cuando tenga que poner las herramientas aritméticas al servicio de la solución de problemas reales en escenarios futuros. A pesar de esto, académicos en el área (Vicente, van Dooren, y Verschaffel, 2014) han establecido que muchos estudiantes pueden no presentar dificultades en la aplicación de operaciones aritméticas básicas, pero estas sí aparecen cuando resuelven problemas realistas. Por ejemplo, Defranco y Curcio (1997) propusieron una situación de división con residuo a un grupo de estudiantes a quienes pidieron encontrar el número de autobuses necesario para transportar un grupo completo a una fiesta. La situación se propuso en dos configuraciones diferentes: en un contexto experimental restrictivo (solo se les presentaba la situación) y un contexto experimental realista (debían hacer la reserva de los autobuses). Los resultados mostraron que 18 de 20 estudiantes fallaron en la configuración experimental restrictiva, al ignorar el residuo de la división y redondear por debajo el número de buses requerido. No obstante, cuando la situación atendió a una configuración experimental realista, 16 de los 20 estudiantes respondieron apropiadamente. Estos resultados sugieren una reducción de las dificultades de los estudiantes cuando las situaciones se plantean en configuraciones realistas, pero no en configuraciones restrictivas, como usualmente sucede en la vida escolar. Silver, Shapiro y Deutsch (1993) reportaron un estudio con una tarea matemática similar en el cual solo $43 \%$ de los participantes no hizo una interpretación apropiada del residuo en la división.

Los resultados obtenidos por Defranco y Curcio (1997) y por Silver, Shapiro y Deutsch (1993) ofrecen información valiosa sobre la formación matemática en la infancia, en particular, con aquellos niños que no han tenido instrucción formal en los algoritmos aritméticos y, en general, con los estudiantes de la escuela primaria para quien urge que la aritmética escolar sea relevante. Esto sugiere que las tareas realistas son aliadas importantes en la formación aritmética en la infancia, particularmente en las tareas de división que requieren una interpretación del residuo. Esta idea se fortalece si se tiene en cuenta la premisa de la Educación Matemática Realista que establece que las matemáticas deberían partir de contextos realistas y centrarse en un conocimiento matemático en la práctica. Los estudiantes necesitan problemas matemáticos realistas que tengan un contexto significativo (Freudenthal, 1991).

En coherencia con las tensiones planteadas, el objetivo de este artículo es proponer algunas reflexiones sobre el rol de las tareas realistas en la interpretación del residuo de la división aritmética y hacer una descripción de las matemáticas que los niños de la escuela primaria ponen en juego en la solución. En ese sentido, la pregunta de investigación que se responde es ¿Cómo contribuyen las tareas realistas en la infancia a la interpretación del residuo en la división aritmética?

\section{MARCO TEÓRICO}

La Educación Matemática Realista es una corriente didáctica que nace en Holanda en los años 60' como respuesta a la enseñanza mecanicista de la aritmética que era común en aquel país. Esta corriente surge en el Instituto para el Desarrollo de la Educación Matemática (IOWO por sus siglas en holandés) — predecesor del Instituto Freu- 
denthal-y busca poner en cuestión el qué y el cómo en la enseñanza de las matemáticas, enfocándose en el progreso del conocimiento de los estudiantes y en su comprensión de las matemáticas. Sus orígenes se remontan a Freudenthal (1991), Gravemeijer (1994), De Lange (1996) y van den Heuvel-Panhuizen (2002), pero en la actualidad varios académicos continúan trabajando en esta línea de pensamiento (Gravemeijer, 2020; Kool, 2020; van den Heuvel-Panhuizen, 2020; Voigt, Fredriksen y Rasmussen, 2020; Vos, 2020).

La Educación Matemática Realista se caracteriza por: (1) el uso privilegiado de problemas contextuales; (2) el desarrollo de modelos como estrategia para el progreso; (3) los aportes de los estudiantes mediante producciones y construcciones propias; (4) la interacción en el proceso de aprendizaje; y (5) el entrelazado de los ejes del currículo (Alsina, 2009; van den Heuvel-Panhuizen, 2002). Esta corriente didáctica plantea que la enseñanza de la matemática debe ser contextual, cercana a los estudiantes y socialmente relevante para que pueda constituirse como actividad y valor humanos (Freudenthal, 1991; van den Heuvel-Panhuizen, 2002). El contexto se convierte en un vehículo para conectar lo concreto con lo abstracto. Lo "realista" no solo hace referencia a la conexión con el mundo real. Esto se aclara al tener en cuenta que la expresión en holandés "zich REALISEren" — de donde la corriente toma su nombre- puede ser traducida como "imaginar" (van den Heuvel-Panhuizen, 2002). En ese sentido, una tarea realista está relacionada con ofrecer a los estudiantes situaciones contextuales que puedan imaginar, es decir, que puedan proyectar en su mente (Sánchez-Barbero, Calatayud y Chamoso, 2019). La expresión "realista" no necesariamente representa un "orden social" como es entendida por Beswick (2011) o un vínculo con la "vida-real" o "cercano a la realidad" como es entendida por Vos (2020); las tareas "realistas" pueden representar una relación con la vida cotidiana, con la fantasía, o con un contexto artificial pero su esencia está en que tengan un significado para los estudiantes y que sean imaginables y recreables. La realidad a la que se refiere Freudenthal no vincula a alguna realidad externa preexistente, sino a la realidad experiencial y autoconstruida (Gravemeijer, 2020); lo que el sentido común experimenta como real (van den Heuvel-Panhuizen, 2002).

La Educación Matemática Realista parte de situaciones contextuales que se usan como mediadores del aprendizaje de las matemáticas que luego son modeladas (usando representaciones, esquemas, diagramas, gráficas, simbolizaciones, ecuaciones, expresiones verbales), dando lugar a la actividad matemática que evoluciona de niveles informales a niveles formales. Esta actividad matemática se conoce con el nombre de matematización. El modelo puede ser simple o sofisticado, pero siempre es emergente y no predeterminado. Primero aparece como un modelo de las estrategias informales de los estudiantes en contextos situados, pero este empieza a transformarse con la actividad matemática y centra su atención en las relaciones matemáticas involucradas. Los modelos evolucionan de un "modelo de" actividad situada a un "modelo para" un razonamiento matemático formal. Como lo expresa Gravemeijer (2020), "el modelo de la actividad matemática informal evoluciona a un modelo para el razonamiento matemático más formal" "(p.223), y esta evolución se da a lo largo de cuatro niveles de actividad: (1) Actividad situada en una tarea que parte de la realidad experiencial para los estudiantes; (2) Actividad referencial, los modelos se refieren a la actividad en la configuración de la tarea; (3) Actividad general, los mo- 
delos se refieren a un marco de relaciones matemáticas; (4) Razonamiento matemático formal, es independiente del soporte de modelos para la actividad matemática.

El proceso de matematización se asocia con la actividad matemática en la cual los estudiantes usan herramientas matemáticas (informales o estándar) para organizar, analizar, dar sentido y solucionar problemas realistas. No obstante, la matematización también se vincula con el proceso de reorganización dentro del sistema matemático mismo, tal como encontrar atajos, descubrir conexiones entre conceptos y estrategias, y luego aplicar esos descubrimientos a otras situaciones (van den Heuvel-Panhuizen, 2002).

Para que la matematización tenga lugar, a los estudiantes se les debe ofrecer tareas contextuales en las que puedan organizar, analizar y elaborar mediante herramientas matemáticas. Las tareas realistas no solo funcionan como un campo de aplicación sino como una fuente para el proceso de aprendizaje. Mientras se trabaja en una tarea realista se desarrollan herramientas matemáticas y comprensión matemática (van den Heuvel-Panhuizen, 2002).

Según Freudenthal (1991), la enseñanza de las matemáticas debe partir de contextos y situaciones problemáticas realistas, en el sentido de que deben ser representables, razonables, imaginables para los estudiantes, como generadores de su actividad matematizadora —organización de la realidad con medios matemáticos-. Así, Freudenthal se refiere al contexto como "ese dominio de la realidad, en el cual, algún proceso de aprendizaje particular es revelado al aprendiz para ser matematizado"2 (p.73).

\section{Metodología}

Para responder a la pregunta de investigación ¿Cómo contribuyen las tareas realistas en la infancia a la interpretación del residuo en la división aritmética?, se acudió a un paradigma cualitativo que busca hacer evidente lo que está oculto y entender cómo los sujetos experimentan el mundo (Creswell, 2013; Denzin y Lincoln, 2012). La interpretación del residuo en la división aritmética es un proceso de naturaleza cualitativo y se puede estudiar a partir de formas en las cuales los participantes le dan sentido, es decir, a partir de una mirada cualitativa. Para tal fin, se diseñó una tarea aritmética realista fundamentada en la Educación Matemática Realista y se implementó con los participantes.

\section{La tarea}

La tarea usada como estrategia para la producción de información fue inspirada en resultados de investigación que han revelado problemas sistemáticos de los estudiantes en situaciones similares (Defranco y Curcio, 1997; Itzcovich, Resia de Moreno, Novembre y Becerril 2009; Silver, Shapiro y Deutsch, 1993). Para resolver la tarea propuesta en esta experiencia, las participantes necesitaban traducir la situación, modelarla, matematizarla e interpretar apropiadamente el residuo. Una tarea similar apareció en el National Assessment of Educational Progress [Evaluación Nacional de Progreso Educativo] (NAEP) en los Estados Unidos (Carpenter, Lindquist, Matthews y Silver, 
1983) y los estudiantes, persistentemente, ignoraron el residuo redondeando los resultados por debajo cuando debían redondear por encima. La tarea en cuestión es la siguiente: Un taxi tiene capacidad para cuatro personas. Diecisiete personas quieren ir a una fiesta ¿cuántos taxis se necesitan para que todas las personas vayan a la fiesta?

En la aplicación de la tarea, el interés no estaba únicamente en el resultado final de la tarea aritmética, sino en todo el proceso de modelado y de matematización que las participantes activaban para resolverla. En ese sentido, la aplicación de la tarea siguió un método dialéctico.

No nos limitamos al método habitual de ofrecer al sujeto estímulos simples a los que esperamos una respuesta directa. [...] De esta manera, podemos estudiar el proceso de llevar a cabo una tarea con la ayuda de medios auxiliares específicos; así también podemos descubrir la estructura interna y el desarrollo de los procesos psicológicos superiores ${ }^{3}$. (Vygotsky, 1983, p.69)

\section{Participantes}

Las participantes de esta experiencia fueron tres estudiantes ( 6 a 10 años) de una misma ciudad, todas de género femenino, estaban matriculadas en el sistema educativo colombiano en el nivel de la básica primaria y - con autorización de sus padres o tutores- aceptaron participar de forma voluntaria. Dos participantes (Valeria y Samanta) avanzaban en el primer periodo (de cuatro posibles) del grado primero y una (Andrea) avanzaba en el primer periodo (de cuatro posibles) en el grado quinto. Valeria y Samanta nunca habían tenido exposición al algoritmo de la división y Andrea era competente en el algoritmo por una y dos cifras. Las participantes no pretenden representar a todos los estudiantes de la escuela primaria, por consiguiente, no se considera una muestra representativa y es necesario tener precaución con las conclusiones. Por el contrario, son una muestra conveniente debido a la disponibilidad y cercanía con la investigadora y así debe ser entendido.

\section{Procedimiento}

La implementación de la tarea se llevó a cabo en un lugar silencioso por fuera del ambiente escolar con cada participante a la vez. Se ofreció a las participantes material manipulativo - en unos casos, cubos de madera de un centímetro de arista y, en otros, fichas de plástico- para que tuvieran oportunidad de usarlos como herramienta de modelado en caso de que fuera necesario y para evidenciar sus estrategias. Toda la acción de las participantes en la resolución de la tarea fue grabada en video que capturaba los gestos, la manipulación del material concreto y las estrategias de organización, modelación y matematización. La interacción entre la investigadora y cada participante fue transcrita palabra a palabra para facilitar el análisis. En la implementación de la tarea se le dio a cada participante la responsabilidad de llegar a sus propias soluciones y explicar su razonamiento. No hubo instrucción directa, solo preguntas de seguimiento y comprobación o preguntas que solicitaban detalles adicionales sobre su razonamiento y acciones: ¿Cómo lo hiciste? ¿Qué quiere decir esto? ¿Entonces cuántos serían? ¿Qué haces con este?

\section{Estrategia de análisis}

Los videos de las participantes resolviendo la tarea realista propuesta se revisaron de forma cuidadosa una y otra vez. Se prestó 
especial atención a los enunciados verbales de las participantes ya que el discurso constituye la conciencia (Lerman 2001) y tiene una función reguladora. También se tuvo en cuenta las acciones de las participantes con los materiales manipulativos como estrategia que ayudara a hacer evidente la actividad matemática. Con los videos se creó una secuencia descriptiva de la actividad matemática y se identificaron episodios cruciales para un análisis adicional. Los episodios cruciales fueron comentados y luego contrastados con el marco teórico. El análisis se centró en diferentes procesos: (1) modelación (2) matematización (3) e interpretación del residuo. El análisis fue inductivo con el objetivo de señalar hallazgos basados en los datos empíricos recolectados (Hernández, 2014). La implementación de la tarea con tres participantes diferentes de forma independiente funcionó como criterio de credibilidad.

\section{Resultados}

A continuación, se presentan los resultados de la implementación de la situación realista con cada una de las participantes. Aparecen en su orden: Valeria, Samanta y Andrea.

\section{Valeria}

A la participante se le presentó la situación realista a resolver junto con diecisiete manipulativos. La participante escuchó las instrucciones y usó los manipulativos para modelar la tarea. Inicialmente, la participante se mostró contrariada con la interpretación que debía dar al residuo de la división, pero la actividad de modelación con los manipulativos le ayudó en una solución creativa de la situación. El siguiente es el intercambio entre la investigadora y la participante.

\begin{tabular}{|c|c|c|}
\hline 1 & Investigadora: & $\begin{array}{l}\text { Un taxi tiene capacidad para cuatro personas. Diecisiete personas quieren ir a } \\
\text { una fiesta ¿Cuántos taxis necesitan para estas personas trasladarse a la fiesta? }\end{array}$ \\
\hline 2 & Valeria: & $\begin{array}{l}\text { [Escucha atentamente la situación planteada y sin mediar palabra procede a } \\
\text { usar el material concreto disponible como apoyo para modelar el escenario en } \\
\text { cuestión. Hace grupos de cuatro como se muestra en la Figura } 1 \text { y queda con } \\
\text { una ficha en su mano] ¿Por qué sobró una? [la participante se hace la pregunta } \\
\text { y piensa por un momento] }\end{array}$ \\
\hline 3 & Valeria: & [después de una extendida pausa] y este que sobró, lo mando en otro taxi \\
\hline 4 & Investigadora: & ¿Solito? \\
\hline 5 & Valeria: & No \\
\hline 6 & Investigadora: & Entonces ¿cuántos taxis necesitas? \\
\hline 7 & Valeria: & Uno más, para que me lleve a este también con todos a la fiesta \\
\hline
\end{tabular}

Figura 1. Disposición del material manipulativo organizado por Valeria.

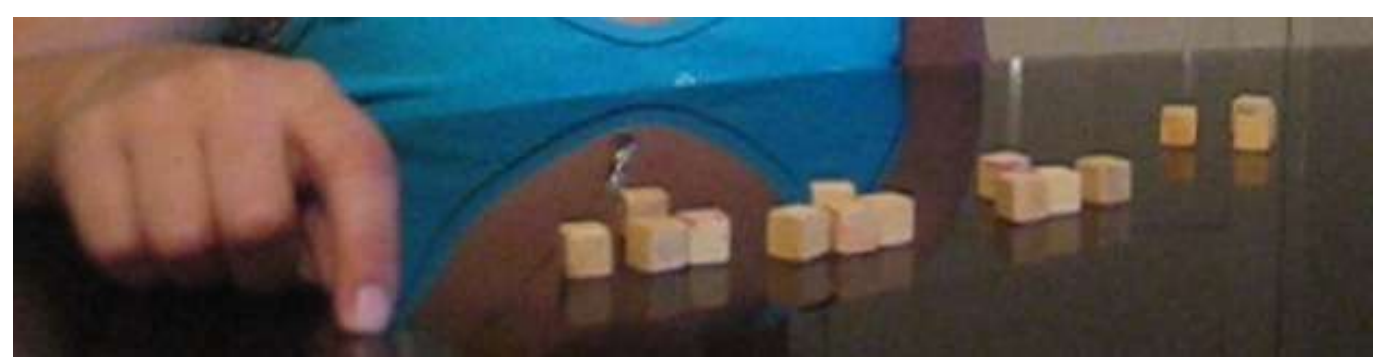


Aunque Valeria no lo dijo de forma explícita, en el video es claro que por cada grupo de cuatro elementos que formó, ella asoció un taxi. Así, sus expresiones "otro taxi" (intercambio 3 en la transcripción) y "uno más" (intercambio 7 en la transcripción) significan un taxi adicional a los cuatro que ya había considerado por cada grupo de cuatro elementos que había formado. Con ello, Valeria necesitó cinco taxis para resolver la situación propuesta. Se resalta que, aunque Valeria no había recibido instrucción en el algoritmo de la división, logró solucionar exitosamente la tarea usando estrategias informales y conocimiento experiencial.

La tarea realista tuvo aportes en el proceso de modelación, en el proceso de matematización y en la interpretación del residuo. Los manipulativos apoyaron el proceso de modelado de la tarea y a llevar un control del reparto equitativo. La ficha que inicialmente le sobró en su reparto ayudó a gestar su pregunta “¿por qué sobro una?” (intercambio 2 en la transcripción). Esta pregunta funcionó como una estrategia de validación de su modelo que posteriormente la llevó a hacer ajustes para darle sentido a la actividad matemática en el contexto de la tarea. También fue evidente el uso de herramientas matemáticas como representación del problema, reparto justo para formar cuatro grupos iguales, adición repetida y relaciones de correspondencia. Su expresión "para que me lleve a este también con todos a la fiesta" (intercambio 7 en la transcripción) indica que Valeria interpretó el residuo en el contexto de la tarea realista.

\section{Samanta}

La situación realista se presentó a la participante junto con diecisiete manipulativos. Samanta no tuvo ninguna duda con respecto a la interpretación del residuo de la división. El siguiente es el intercambio entre investigadora y participante.

\begin{tabular}{lll}
\hline 8 & Investigadora: & $\begin{array}{l}\text { Tienes diecisiete personas que quieren ir a una fiesta, y quieren ir en taxi. } \\
\text { Cada taxi tiene capacidad para cuatro personas ¿Cuántos taxis necesitas } \\
\text { para que todas las personas vayan a la fiesta? }\end{array}$ \\
\hline 9 & Samanta: & $\begin{array}{l}\text { [Usa las fichas para modelar la situación. Hace grupos de cuatro fichas } \\
\text { como aparece en la Figura 2] }\end{array}$ \\
\hline 10 & Investigadora: & ¿Qué quiere decir esto? [señalando uno de los grupos de cuatro] \\
\hline 11 & Samanta: & Que hay cuatro personas en el taxi y una en la parte de adelante \\
\hline 12 & Investigadora: & Ah, comprendo \\
\hline 13 & Investigadora: & [Después de una pausa] ¿Entonces cuántos taxis van acá? \\
\hline 14 & Samanta: & $\begin{array}{l}\text { [Cuenta cada uno de los grupos de cuatro y cuenta también la ficha indivi- } \\
\text { dual] uno, dos, tres, cuatro y cinco. }\end{array}$ \\
\hline
\end{tabular}

Figura 2. Disposición del material manipulativo por parte de Samanta.

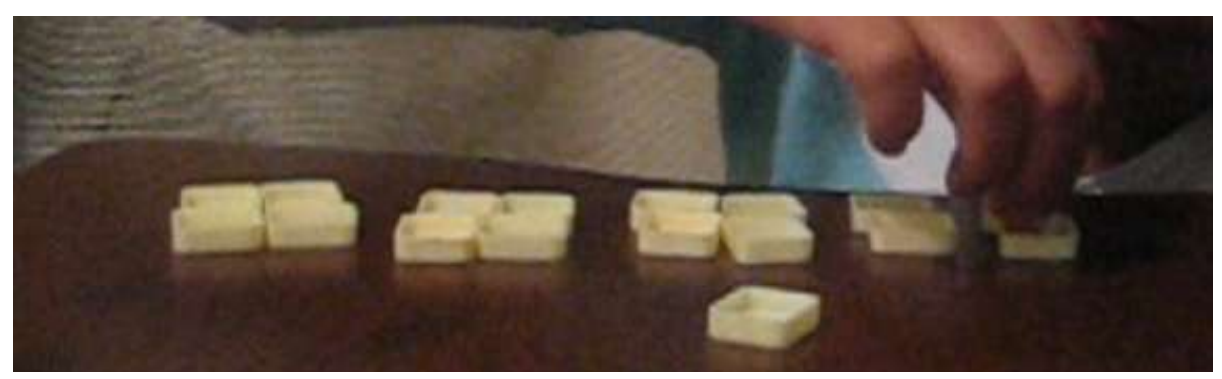


En el desarrollo de la tarea se evidenció que Samanta usó repartos equitativos como estrategia para encontrar la solución. Cuando la participante expresó "hay cuatro personas en el taxi y una en la parte de adelante" (intercambio 11 en la transcripción), señaló uno de los grupos que había formado con cuatro elementos del material manipulativo y usó su conocimiento no matemático referido a un taxi. En su ciudad, un taxi típico tenía capacidad para transportar cuatro personas: una en la parte delantera y tres en la parte trasera.

Así, ella vinculó este conocimiento experiencial —no matemático- para dar sentido a la situación. Con la expresión "hay cuatro personas en el taxi y una en la parte de adelante" (intercambio 11 en la transcripción), Samanta también comunicó su interpretación de la tarea y la traducción que hizo de la situación contextual al mundo matemático y dio a entender su estrategia de modelación. Al final, cuando contó hasta cinco (intercambio 14 en la transcripción), Samanta aplicó una relación de correspondencia donde a cada grupo de cuatro elementos $-\mathrm{y}$ al elemento solitario- le asignó un taxi. Este ejercicio de correspondencia, al igual que con la adición repetida y el conteo, es una herramienta matemática que hace evidente el proceso de matematización. Estas surgieron como actividad situada en la experiencia de la participante, a un nivel elemental, pero se consideran como precursoras de un desarrollo más formalizado de la división aritméti- ca. Al terminar el modelado de la situación realista con el material manipulativo y poner en juego herramientas matemáticas, Samanta no tuvo dudas al considerar el residuo de la división como elemento de esa relación de correspondencia. Ella interpretó el residuo de ese reparto en términos de la situación realista. El hecho de considerar un taxi para el pasajero solitario da cuenta de ello.

\section{Andrea}

Se le presentó la situación realista a Andrea - la participante de nivel académico más alto- además de diecisiete manipulativos para usar en caso de ser necesario. Cuando se enfrentó a resolver la situación por primera vez, el proceso de matematización sucedió mentalmente y no fue verbalizado. La investigadora no pudo observar las estrategias que tuvieron lugar allí porque la participante, aunque usó los manipulativos dispuestos, no dejó ver una clara organización en su disposición final (Figura 3). A pesar de que la estrategia con los manipulativos no fue suficientemente clara, la participante señaló que se necesitaban cuatro taxis. La investigadora tuvo que interrogar a la participante para que ofreciera detalles sobre las estrategias de modelado y matematización. En esta nueva oportunidad, Andrea usó los manipulativos para explicar su estrategia, se dio cuenta de su error y ajustó su respuesta inicial a cinco taxis. El siguiente es el intercambio entre la investigadora y la participante.

Figura 3. Disposición inicial de los manipulativos por parte de Andrea.

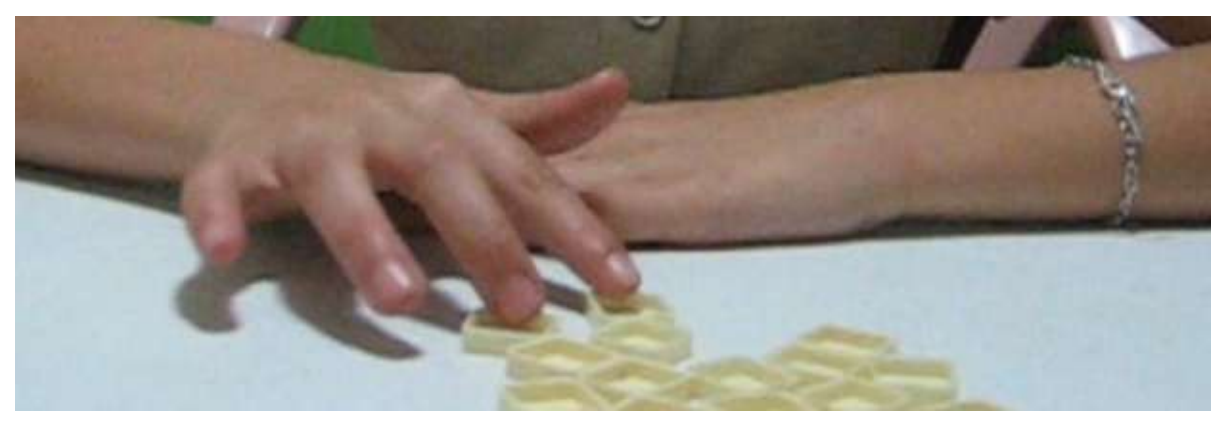




\begin{tabular}{lll}
\hline 15 & Investigadora: & $\begin{array}{l}\text { Diecisiete personas van a ir a una fiesta, van a ir en taxi. Cada taxi } \\
\text { tiene capacidad para cuatro personas ¿Cuántos taxis se necesitan? }\end{array}$ \\
\hline 16 & Andrea: & $\begin{array}{l}\text { [Usa los manipulativos, pero no es clara su estrategia. Figura 3] Cua- } \\
\text { tro }\end{array}$ \\
\hline 17 & Investigadora: & Cuéntame ¿cómo lo hiciste? \\
\hline 18 & Andrea: & $\begin{array}{l}\text { Sumé cuatro, más cuatro, más cuatro, más cuatro y así. Por } \\
\text { ejemplo [toma las fichas y hace grupos de cuatro elementos. Figura } \\
\text { 4], cuatro serían un taxi, otros cuatro, dos taxis, otros cuatro tres, } \\
\text { taxis, y otros cuatro, cuatro taxis. Sobraría una [la participante } \\
\text { hace un gesto que da a entender que hay algo extraño en su } \\
\text { razonamiento que no la deja completamente satisfecha]. }\end{array}$ \\
\hline 19 & Investigadora: & ¿Entonces qué haces con esa persona? \\
\hline 20 & Andrea: & Tengo que conseguir otro taxi \\
\hline 21 & Investigadora: & ¿Entonces cuántos taxis serían? \\
\hline 22 & Andrea: & Cinco \\
\hline
\end{tabular}

Figura 4. Disposición de los manipulativos por parte de Andrea después de ser interrogada.

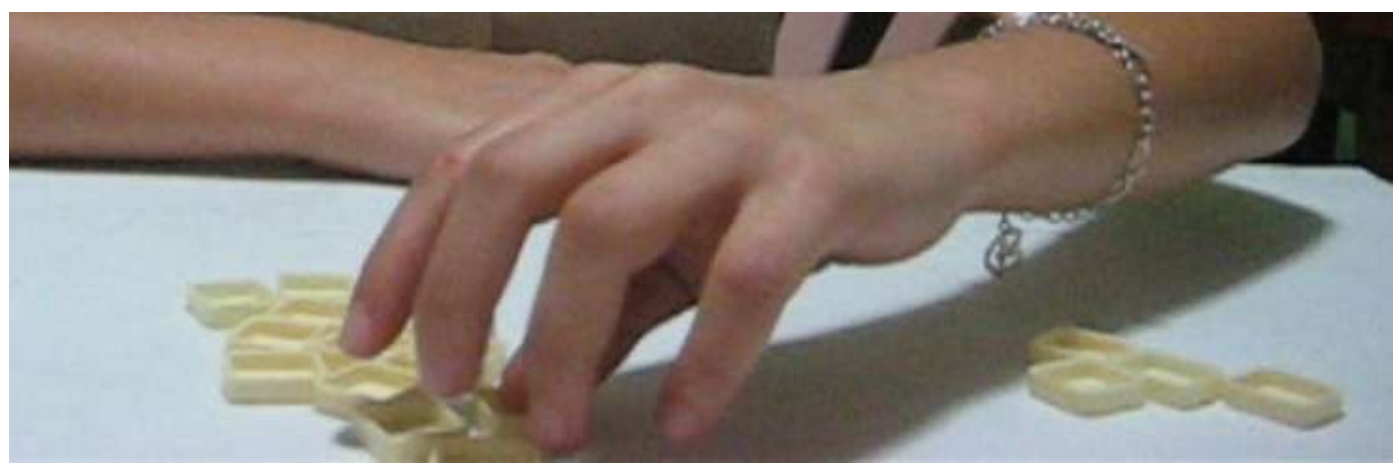

Aunque Andrea era la única participante con experiencia en el algoritmo de la división, su primera respuesta se basó en el uso mental de sumas repetidas y hechos numéricos que la llevaron a un resultado errado en el que interpretó equivocadamente el residuo. Cuando la investigadora le pidió explicar su razonamiento modeló con los manipulativos formando cuatro grupos de cuatro y un elemento solitario. Esta nueva representación le permitió ver físicamente el residuo y otorgarle una interpretación en el contexto de la tarea. Andrea ajustó su respuesta inicial y expresó “Tengo que conseguir otro taxi” (inter- cambio 20 en la transcripción). Andrea usó herramientas como: traducción de la tarea del mundo real al mundo matemático, adición repetida, hechos numéricos, relación de correspondencia entre cada grupo de cuatro (y el elemento solitario) con un taxi. Estas herramientas hacen evidente el proceso de matematización que tuvo lugar. En ese proceso interactivo de modelado y matematización, Andrea tuvo la oportunidad de explicar su respuesta, reflexionar y hacer los ajustes que revelaron su avance. Modelado y matematización funcionaron como puentes entre la tarea contextual y la actividad matemática. 


\section{Discusión}

El trabajo con manipulativos se vio representado en las soluciones a la tarea realista que dieron Valeria y Samanta. Andrea sólo usó los manipulativos cuando fue cuestionada por la investigadora y era la única participante con instrucción formal en el algoritmo de la división; no obstante, no usó el algoritmo formal sino otras habilidades informales para encontrar la respuesta inicial. Al respecto van den Heuvel-Panhuizen (2002) sostiene que "los contextos que tienen potencial para el modelado funcionan como potentes dispositivos para cerrar la brecha entre las matemáticas informales y las más formales ${ }^{4}$ (p.11). Solo cuando Andrea usó los manipulativos para explicar su solución, representó la situación con un modelo - cuatro grupos de cuatro y un elemento solitario- que le permitió reconocer su error y ajustar su respuesta. Allí, el modelo tuvo la función de validación que le permitió mejorar su cálculo e interpretación. Al respecto, van den Heuvel-Panhuizen (2002) reconoce que los modelos se originan de situaciones que funcionan como puentes a niveles más elevados de comprensión. En este mismo sentido, Gravemeijer (2020) sostiene que los modelos visuales y táctiles han sido poderosas herramientas para apoyar el aprendizaje para la comprensión. Este es un hallazgo en favor de los materiales manipulativos para la modelación. Aún para infantes que tengan un razonamiento mental interiorizado (como el de Andrea), los materiales manipulativos son una herramienta de modelado que apoya en la explicación, justificación, ejemplificación, validación y confrontación del razonamiento. Aunque el propósito de la Educación Matemática Realista es que los estudiantes usen el modelado para avanzar en los diferentes niveles de actividad matemática, el uso de los manipulativos se configura como una herramienta esencial en ese modelado.

La tarea realista con el apoyo de los manipulativos también ofreció oportunidades de matematización. Las participantes pusieron en juego tanto herramientas matemáticas informales como formales: conteo, reparto justo, adición repetida, hechos numéricos y relaciones de correspondencia. El apoyo de los manipulativos hizo evidente las relaciones de correspondencia que de otra forma no hubiese sido posible; todas estas herramientas fueron situadas en la tarea matemática. Es de resaltar que la tarea realista y los manipulativos fueron claves para que las herramientas matemáticas que emergieron para la representación, análisis y solución tuvieran un sentido en términos del contexto. Se hace claro el llamado de algunos autores a ver las matemáticas integradas en la actividad matemática: "el punto focal no debería estar en las matemáticas como un sistema cerrado sino en la actividad, en el proceso de matematización"5 (van den Heuvel-Panhuizen, 2002, p.3). La tarea realista también permitió evidenciar diferentes niveles de matematización. Mientras Valeria y Samanta usaron herramientas matemáticas básicas dentro de la actividad situada, Andrea usó herramientas más sofisticadas usando relaciones matemáticas. Así, la tarea realista impulsó diferentes niveles de matematización. Al respecto es relevante destacar el pensamiento de van den Heuvel-Panhuizen (2002) quien establece que las tareas realistas no sólo funcionan como un campo de aplicación, sino como una fuente de progreso para el aprendizaje. Mientras se trabaja en una tarea realista se pueden desarrollar herramientas y comprensión matemática. En un sentido similar, la utilización de dife- 
rentes herramientas matemáticas en la solución de una tarea hace posible múltiples modos de matematizar las situaciones usando diferentes modelos y lenguajes (de Lange, 1996; Gravemeijer, 1994). Así mismo, se ha destacado el valor de las estrategias matemáticas informales para encontrar una solución exitosa a una tarea realista, las cuales se consideran herramientas que dan cuenta del proceso de matematización (Gravemeijer, 2020).

El problema del residuo en los problemas de división aritmética ha sido ampliamente estudiado (Carpenter, Lindquist, Matthews y Silver, 1983; Defranco y Curcio, 1997; Silver, Shapiro y Deutsch, 1993) evidenciando coincidencias en las dificultades de los participantes al aportar una interpretación coherente. Al respecto, las tareas realistas parecen ser escenarios propicios para aumentar las posibilidades de otorgar una interpretación más contextual. Expresiones como "Uno más, para que me lleve a este también con todos a la fiesta" (intercambio 7 en la transcripción) y "Tengo que conseguir otro taxi" (intercambio 20 en la transcripción), sugieren que las participantes interpretaron el resultado en términos del contexto. Esto coincide con declaraciones de autores como van den Heuvel-Panhuizen (2002), quien resalta que el contexto dota de significado al resultado. Esto se torna aún más llamativo si se tiene en cuenta las participantes que no habían recibido instrucción formal en el algoritmo de la división. Ellas usaron las herramientas disponibles - conteos, repartos equitativos, sumas repetidas, conocimiento experiencial- para solucionar y dar sentido a la tarea realista y activaron sus recursos para aproximarse a la división como un reparto e interpretar el residuo en términos contextuales.
Los resultados de esta experiencia sugieren una perspectiva diferente en comparación con estudios anteriores que han establecido que es poco probable que los niños tengan éxito en la división si no tienen una buena comprensión de los conceptos subyacentes a las habilidades de multiplicación (Cisternas Rojas, Gil Llario, Ceccato, y Marí-Sanmillán, 2019) y que la comprensión de la división requiere una buena comprensión de la multiplicación (Kaasila, Pehkonen y Hellinen, 2010). En un sentido argumentativo similar:

Los clásicos de la literatura psicológica, como las obras de Binet y otros, suponen que el desarrollo es siempre un requisito previo para el aprendizaje y que si las funciones mentales (operaciones intelectuales) de un niño no han madurado en la medida en que es capaz de aprender un tema particular, entonces ninguna instrucción resultará útil. Temían especialmente la instrucción prematura, la enseñanza de un tema antes de que el niño estuviera listo para ello. Todo el esfuerzo se concentró en encontrar el umbral más bajo de la capacidad de aprendizaje, la edad a la cual un tipo particular de aprendizaje se hace posible por primera vez 6 . (p.72)

Esta experiencia mostró que aun sin haber desarrollado el algoritmo formal de la división, las participantes lograron dar solución satisfactoria a la tarea propuesta en un escenario realista. En contraste, los hallazgos de esta experiencia confirman que "los niños tienen su propia aritmética preescolar" (Vygotsky, 1983, p.76). Es decir, los niños en sus experiencias previas a la vida escolar desarrollan ideas matemáticas como el sentido de número (van den Heuvel-Panhuizen y van den Boogaard, 2008) y el sentido de reparto justo que usan como herramientas informales en su actividad matemática. Al 
respecto, la escuela debe potenciar estas experiencias previas para favorecer el desarrollo espontáneo que posteriormente den lugar a los procesos emergentes más formalizados de modelación y matematización.

\section{CONCLUSIONES Y LIMITACIONES}

Las reflexiones originadas en esta experiencia mostraron el potencial de los contextos realistas en la infancia en la interpretación del residuo de la división aritmética. También se hicieron evidentes los procesos de modelación y matematización que tienen lugar cuando los estudiantes de la escuela primaria dan soluciones creativas a situaciones realistas.

Los resultados de esta experiencia permiten sugerir que las tareas matemáticas realistas - como la propuesta aquí- con apoyo de materiales manipulativos (1) podrían ser escenarios para que los estudiantes exploren, construyan e implementen algoritmos espontáneos; (2) parecen solucionar el problema de la interpretación del residuo en la división aritmética; (3) podrían funcionar como promotoras del modelado y la matematización dando acceso a una matemática mucho más formal.

Una limitación de esta experiencia es el número reducido de participantes. Como se indicó en el componente metodológico, las participantes fueron una muestra conveniente $\mathrm{y}$, por lo tanto, los juicios derivados de ella deben ser formulados con precaución. Sería interesante llevar a cabo esta experiencia con un número mayor de participantes que sean representativos de la básica primaria para determinar si los hallazgos son consistentes.

Otra limitación de esta experiencia es que se llevó a cabo por fuera del ambiente escolar, en un ambiente artificial que no se asemeja a la realidad de la escuela. No obstante, los hallazgos sugieren que las situaciones que se presentan en contextos realistas tienen el potencial para poner en juego matemáticas espontáneas de los participantes y para activar recursos matemáticos que no estaban disponibles. Esto es, las situaciones dentro de un contexto realista pueden convertirse de forma eventual en la puerta de acceso a un conocimiento matemático formal. La investigación futura podría explorar variadas situaciones realistas en ambientes escolares y contrastar la consistencia con los hallazgos presentados en esta experiencia.

Otra limitación tiene que ver con la naturaleza transversal de esta experiencia. Un corte transversal solo describe el fenómeno de interés en un momento en particular. Es claro que el propósito de la Educación Matemática Realista es de naturaleza longitudinal en el sentido que el estudiante, al enfrentarse a diferentes situaciones contextuales, se expone a múltiples oportunidades para poner en acción el conocimiento matemático intencionado y para el progreso matemático. En consecuencia, la investigación en educación matemática necesita robustecer el conocimiento científico con estudios de naturaleza longitudinal que puedan mostrar la historia completa del uso de la matemática realista en la infancia y de la emergencia del modelado y la matematización en la división aritmética. 


\section{Notas}

1. Traducción de la autora. "The model of informal mathematical activity develops into a model for more formal mathematical reasoning."

2. Traducción de la autora. "Contexts then means that domain of reality, which in some particular learning process is disclosed to the learner in order to be mathematised."

3. Traducción de la autora. "We do not limit ourselves to the usual method of offering the subject simple stimuli to which we expect a direct response. [...] In this way, we are able to study the process of accomplishing a task by the aid of specific auxiliary means; thus we are also able to discover the inner structure and development of the higher psychological processes."

4. Traducción de la autora. "Contexts that have model potential serve as an important device for bridging this gap between informal and more formal mathematics."

5. Traducción de la autora. "The focal point should not be on mathematics as a closed system but on the activity, on the process of mathematization."

6. Traducción de la autora. "Similarly, the classics of psychological literature, such as the works by Binet and others, assume that development is always a prerequisite for learning and that if a child's mental functions (intellectual operations) have not matured to the extent that he is capable of learning a particular subject, then no instruction will prove useful. They especially feared premature instruction, the teaching of a subject before the child was ready for it. All effort was concentrated on finding the lower threshold of learning ability, the age at which a particular kind of learning first becomes possible."

\section{REFERENCIAS}

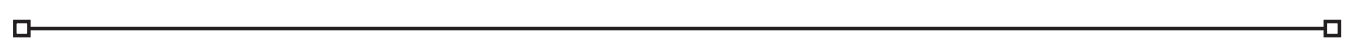

Alsina, A. (2009). El aprendizaje realista: una contribución de la investigación en Educación Matemática a la formación del profesorado. En M. J. González, M. T. González \& J. Murillo (Eds.), Investigación en Educación Matemática XIII (pp. 119 - 127). Santander: SEIEM.

Bakker, A., \& Derry, J. (2011). Lessons from Inferentialism for Statistics Education [Lecciones del inferencialismo para la educación estadística]. Mathematical Thinking and Learning, 13(1-2), 5-26. doi: 10.1080/10986065.2011.538293

Beswick, K. (2011). Putting context in context: an examination of the evidence for the benefits of 'contextualised' tasks. International Journal of Science and Mathematics Education 9, 367-390.

Carpenter, T. P., Lindquist, M. M., Matthews, W., \& Silver, E. A. (1983). Results of the third NAEP mathematics assessment: Secondary school. Mathematics Teacher, 76, 652-659. 
Cisternas Rojas, Y., Gil Llario, M. D., Ceccato, R., y Marí-Sanmillán. (2019). Analysis of the psychometric properties of a multiplication and division processes assessment scale. International Journal of Developmental and Educational Psychology, 1 - Monográfico 2, 159-166.

Creswell, J. W. (2013). Research design qualitative, quantitative, and mixed methods approaches. Los Angeles: Sage.

Defranco, T. C., \& Curcio, F. R. (1997). A division problem with a remainder embedded across two contexts: Children's solutions in restrictive versus real-world settings. Focus on Learning Problems in Mathematics, 19 (2), 58-72.

De Lange, J. (1996). Using and applying mathematics in education. En A.J. Bishop (Ed). International Handbook of Mathematics Education, Part I (pp. 49-97). Utrecht: Kluwer Academia Press.

Denzin, N. K., \& Lincoln, Y. (2012). La investigación cualitativa como disciplina y como práctica. En N. K. Denzin, \& Y. Lincoln (Edits.), Manual de investigación cualitativa, vol. 1: El campo de la investigación cualitativa (pp. 43-102). Barcelona: Gedisa.

Freudenthal, H. (1991). Revisiting mathematics education. Dordrectht: Kluwer Academic Publishers.

Gravemeijer, K (1994). Developing realistic mathematics education. Utrecht: Freudenthal Institute.

Gravemeijer, K. (2020). A socio-constructivist elaboration of Realistic Mathematics Education. En M. Van den Heuvel-Panhuizen (ed.), National Reflections on the Netherlands Didactics of Mathematics, ICME-13 Monographs, (pp. 217-233). Springer https://doi.org/10.1007/978-3-03033824-4_12

Hernández, R. (2014). Qualitative research through interviews: Its analysis by Grounded Theory. Cuestiones Pedagógicas, 23, 187-210.

Itzcovich, H., Resia de Moreno, B., Novembre, A., \& Becerril, M. M. (2009). La matemática escolar: las prácticas de enseñanza en el aula. Buenos Aires: AIQUE.

Kaasila, R., Pehkonen, E. \& Hellinen, A. (2010). Finnish pre-service teachers' and upper secondary students' understanding of division and reasoning strategies used. Educational Studies in Mathematics, 73 (3) 247-261.

Kool, M. (2020). Sixteenth century reckoners versus twenty-first century problem solvers. En M. Van den Heuvel-Panhuizen (ed.), National Reflections on the Netherlands Didactics of Mathematics, ICME-13 Monographs, (pp.105-120). Springer https://doi.org/10.1007/978-3-030-33824-4_7

Lerman, S. (2001). Cultural, discursive psychology: a sociocultural approach to studying the teaching and learning of mathematics. Educational Studies in Mathematics, 46, 87-113.

Ministerio de Educación Nacional. (2006). Estándares Básicos de Competencias en Lenguaje, Matemáticas, Ciencias y Ciudadanas. Bogotá: Revolución Educativa Colombia Aprende MEN.

National Governors Association \& Council of Chief State School Officers. (2010). Common Core State Standards Initiative. Washington, DC: NGA and CCSSO. 
Sánchez-Barbero, B; Calatayud, M., y Chamoso, J. M. (2019). Análisis de la interacción de maestros cuando resuelven problemas realistas conjuntamente con sus alumnos en aulas de primaria, teniendo en cuenta su experiencia docente. Uni-pluriversidad, 19(2), 40-59. doi: 10.17533/udea. unipluri.19.2.03

Silver, E., Shapiro, L., \& Deutsch, A. (1993). Sense making and the solution of division problems involving remainders: An examination of middle school students' solution processes and their interpretations of solutions. Journal for Research in Mathematics Education, 24(2), 117-135.

Van den Heuvel-Panhuizen, M. (2002). Realistic Mathematics Education as work in progress. En Fou-Lai Lin (Eds.). Common sense in mathematics education. Proceedings of 2001 The Netherlands and Taiwan Conference on Mathematics Education (pp. 1-43). Taiwan: National Taiwan Normal University.

Van den Heuvel-Panhuizen (2020). A spotlight on Mathematics Education in the Netherlands and the central role of Realistic Mathematics Education. En M. Van den Heuvel-Panhuizen (ed.), National Reflections on the Netherlands Didactics of Mathematics, ICME-13 Monographs, (pp. 1-14) https://doi.org/10.1007/978-3-030-33824-4_1

Van den Heuvel-Panhuizen, M., \& van den Boogaard, S. (2008). Picture Books as an Impetus for Kindergartners' Mathematical Thinking. Mathematical Thinking and Learning, 10(4) 341-373, DOI: $10.1080 / 10986060802425539$

Vicente, S., van Dooren, W., \& Verschaffel (2014). Utilizar las matemáticas para resolver problemas reales. Cultura y Educación, 20 (4) 391-406, DOI:10.1174/113564008786542235

Voigt, M., Fredriksen, H., Rasmussen, C. (2020). Leveraging the design heuristics of realistic mathematics education and culturally responsive pedagogy to create a richer flipped classroom calculus curriculum. ZDM. https://doi.org/10.1007/s11858-019-01124-х

Vos, P. (2020). Task contexts in Dutch Mathematics Education. En M. Van den Heuvel-Panhuizen (ed.), National Reflections on the Netherlands Didactics of Mathematics, ICME-13 Monographs, (pp. 31-53). Springer https://doi.org/10.1007/978-3-030-33824-4_3

Vygotsky, L. (1983). Pensamiento y lenguaje: Teoría del desarrollo cultural y las funciones psíquicas. Buenos Aires: La Pleyade.

Zapata-Cardona, L. (2018). Enseñanza de la estadística desde una perspectiva crítica. Yupana, 10, $30-41$.

Zapata-Cardona, L. \& Marrugo, L. (2019). Critical citizenship in Colombian statistics textbooks. En G. Burrill, \& D. Ben-Zvi (Eds.), Topics and Trends in Current Statistics Education Research. International Perspectives (pp. 373-389). Switzerland: Springer. 